\title{
Molecular Correlates and Nuclear Features of Encapsulated Follicular-Patterned Thyroid Neoplasms
}

Chan Kwon Jung ${ }^{1}$, Andrey Bychkov², Dong Eun Song ${ }^{3}$, Jang-Hee Kim, Yun Zhu ${ }^{4}$ Zhiyan Liu ${ }^{6}$, Somboon Keelawat ${ }^{7}$, Chiung-Ru Lai ${ }^{8}$, Mitsuyoshi Hirokawa ${ }^{9}$, Kaori Kameyama ${ }^{10}$, Kennichi Kakudo ${ }^{11}$

${ }^{1}$ Department of Hospital Pathology, College of Medicine, The Catholic University of Korea, Seoul, Korea; ${ }^{2}$ Department of Pathology, Kameda Medical Center, Kamogawa, Japan; ${ }^{3}$ Department of Pathology, Asan Medical Center, University of Ulsan College of Medicine, Seoul; ${ }^{4}$ Department of Pathology, Ajou University School of Medicine, Suwon, Korea; ${ }^{5}$ Department of Pathology, Jiangsu Institute of Nuclear Medicine, Wuxi; ${ }^{6}$ Department of Pathology, Sixth People's Hospital, Shanghai Jiao Tong University, Shanghai, China; ${ }^{7}$ Department of Pathology, Faculty of Medicine, Chulalongkorn University, Bangkok, Thailand; ${ }^{8}$ Department of Pathology, Taipei Veterans General Hospital, Taipei, Taiwan; ${ }^{9}$ Department of Diagnostic Pathology and Cytology, Kuma Hospital, Kobe; ${ }^{10}$ Division of Diagnostic Pathology, Keio University Hospital, Tokyo; ${ }^{11}$ Department of Pathology and Thyroid Disease Center, Izumi City General Hospital, Izumi, Japan

Background: Assessing nuclear features is diagnostically challenging in the aspect of thyroid pathology. The aim of this study was to determine whether pathologists could distinguish $B R A F$-like and $R A S$-like nuclear features morphologically and identify morphological features to differentiate thyroid tumors with $R A S$-like mutations from encapsulated papillary thyroid carcinoma (PTC) with predominant follicular growth and $B R A F^{\mathrm{V} 600 \mathrm{E}}$ mutation.

Methods: Representative whole slide images of 16 encapsulated thyroid tumors with predominant follicular growth were reviewed by 12 thyroid pathologists using a web browser-based image viewer. Total nuclear score was calculated from semi-quantitatively scored eight nuclear features. The molecular profile of $R A S$ and $B R A F$ genes was determined by Sanger sequencing.

Results: Total nuclear score ranging 0 to 24 could differentiate $B R A F$-like tumors from $R A S$-like tumors with a cut-off value of score 14. The interobserver agreement was the highest for the assessment of nuclear pseudoinclusions (NPIs) but the lowest for nuclear elongation and sickle-shaped nuclei. NPIs were found in tumors with $B R A F^{\mathrm{V} 600 \mathrm{E}}$ mutation, but not in tumors with $R A S$-like mutations. Total nuclear scores were significantly higher for tumors with $B R A F^{\mathrm{V} 600 \mathrm{E}}$ than for those with $R A S$-like mutations $(P<0.001)$. Conclusion: Our results suggest that NPIs and high nuclear scores have diagnostic utility as rule-in markers for differentiating PTC with $B R A F^{\mathrm{V} 600 \mathrm{E}}$ mutation from benign or borderline follicular tumors with $R A S$-like mutations. Relaxation of rigid criteria for nuclear features resulted in an overdiagnosis of PTC. Immunostaining or molecular testing for $B R A F^{\mathrm{V} 600 \mathrm{E}}$ mutation is a useful adjunct for cases with high nuclear scores to identify true PTC.

Keywords: Thyroid cancer, papillary; Proto-oncogene proteins B-raf; Genes, ras; Thyroid neoplasms; Mutation; Medical overuse; Pathologists; Observer variation

Received: 1 October 2020, Revised: 7 November 2020, Accepted: 14 January 2021

Corresponding author: Chan Kwon Jung

Department of Pathology, Seoul St. Mary's Hospital, College of Medicine, The Catholic University of Korea, 222 Banpo-daero, Seocho-gu, Seoul 06591, Korea Tel: +82-2-2258-1622, Fax: +82-2-2258-1627, E-mail: ckjung@catholic.ac.kr
Copyright $\odot 2021$ Korean Endocrine Society

This is an Open Access article distributed under the terms of the Creative Commons Attribution Non-Commercial License (https://creativecommons.org/ licenses/by-nc/4.0/) which permits unrestricted non-commercial use, distribution, and reproduction in any medium, provided the original work is properly cited. 


\section{INTRODUCTION}

Encapsulated follicular-patterned thyroid tumors are commonly encountered in thyroid cytopathology/histopathology practice. They include benign (follicular adenoma), borderline (noninvasive follicular thyroid neoplasm with papillary-like nuclear features [NIFTP], well-differentiated tumor of uncertain malignant potential, follicular tumor of uncertain malignant potential), and malignant (follicular variant of papillary thyroid carcinoma [PTC], follicular thyroid carcinoma, well-differentiated carcinoma, not otherwise specified) tumors [1]. Pathologic diagnosis of these lesions can be made based on the histologic examination of capsule (capsular/vascular invasion) and nuclear features of PTC. These tumors frequently pose diagnostic difficulty to pathologists because of their overlapping morphologic features and poor interobserver agreement for their diagnosis $[2,3]$.

NIFTP is a borderline tumor that shows encapsulation or well demarcation, follicular growth pattern, and PTC-type nuclear changes $[4,5]$. According to revised diagnostic criteria for NIFTP, exclusion criteria include the presence of invasion, true papillae, psammoma bodies, solid/trabecular growth more than $30 \%$, and $B R A F^{\mathrm{V} 600 \mathrm{E}}$ or TERT promoter mutations [5]. Given that NIFTP should qualify stringent diagnostic criteria and that is a $R A S$-like tumor, it is important to reveal microscopic features helpful for distinguishing $R A S$-like tumors from PTC with $B R A F^{\mathrm{V} 600 \mathrm{E}}$ mutation when molecular data are unavailable.

Morphological and biological characteristics of $R A S$-like thyroid tumors are much different from those of $B R A F^{\mathrm{V} 600 \mathrm{E}}$-mutated thyroid tumors [6]. RAS and other RAS-like mutations $\left(B R A F^{\mathrm{K} 601 \mathrm{E}}\right.$, eukaryotic translation initiation factor 1A X-linked [EIFIAX] mutations, peroxisome proliferator-activated receptor gamma $[P P A R G]$ fusion, thyroid adenoma associated [THADA] fusion, and so forth) have been found in various types of thyroid tumors with follicular growth pattern ranging from follicular adenoma to follicular carcinoma whereas $B R A F^{\mathrm{V} 600 \mathrm{E}}$ mutation is predominantly found in PTCs with papillary architecture and in advanced thyroid cancers, such as poorly differentiated and anaplastic thyroid carcinomas [6-8].

A three-point nuclear scoring scheme was developed by the working group for the reevaluation of the encapsulated follicular variant of PTC [4]. Three categories of nuclear features include nuclear size and shape, nuclear membrane irregularities, and chromatic characteristics. When a category is present at a significant level, it is assigned as a value of 1 . When each category is absent or only slightly expressed, its value is 0 . Therefore, the summation nuclear score ranges from 0 to 3 . Nuclear features of PTC with a nuclear score of 2 or 3 are required for the diagnosis of PTC and NIFTP $[1,4]$. However, molecular evidence has revealed that thyroid tumors with nuclear score of 2 are distinct from those with nuclear score of 3 [5]. Most PTCs with $B R A F^{\mathrm{V} 600 \mathrm{E}}$ show a nuclear score of 3 whereas RAS-mutated PTC and NIFTP usually have a nuclear score of 2 [5,9]. Tumors with $R A S$-like mutations usually show mild nuclear enlargement, mild irregularity of nuclear membranes, delicate nuclear grooves, and slightly pale chromatin whereas nuclear pseudoinclusions (NPIs) are absent or rarely seen [10]. Recent studies have reported that for cases of noninvasive encapsulated thyroid tumors, Asian pathologists, especially those in Japan, are more stringent in making a diagnosis of PTC than Western pathologists [11-15]. Therefore, noninvasive follicular tumors with a nuclear score of 2 are more frequently diagnosed as follicular adenomas by Asian pathologists whereas the same tumors are often classified as NIFTP or PTC in Western practice [13-16]. Such diagnostic discrepancies contributed to observed differences in prevalence rates of PTC and NIFTP across countries $[13,14]$.

The objective of the present study was to clarify whether pathologists could distinguish $B R A F$-like and $R A S$-like nuclear features morphologically. Another aim of the present study was to establish reliable diagnostic criteria to identify $B R A F$-like and $R A S$-like tumors in noninvasive encapsulated follicular-patterned neoplasms.

\section{METHODS}

This retrospective study was approved by the Institutional Review Board of the Catholic University of Korea, Seoul St. Mary's Hospital (KC16SISI0709). Informed consent was waived by the board. Cases were selected with a stratified two-stage cluster sampling. Out of 40 encapsulated follicular-derived tumors with predominant follicular growth (less than $1 \%$ papillae) initially selected as having equivocal nuclear features of PTC, 16 cases with available molecular workup were enrolled in this study.

All cases had either $B R A F^{\mathrm{V} 600 \mathrm{E}}, B R A F^{\mathrm{K} 601 \mathrm{E}}$, or mutation of RAS (NRAS, HRAS, and KRAS) at codon 61. Molecular profile was determined by Sanger sequencing, as previously described [17, 18]. Tumors were classified as $B R A F$-like $\left(B R A F^{\mathrm{V} 600 \mathrm{E}}\right)$ and $R A S$ like ( $B R A F^{\mathrm{K} 601 \mathrm{E}}$ or $R A S$ mutations). All histological slides were stained with hematoxylin and eosin. One representative section per case was digitally scanned at $40 \times$ using a Pannoramic MIDI II slide scanner (3DHistech, Budapest, Hungary). 


\section{Observers}

This study was initially opened to all members of the Asian Working Group in Thyroid Pathology [19,20], enrolling volunteers who had a special interest and expertise in thyroid pathology. Twelve thyroid pathologists with 8 to 40 years of experience from 12 centers located in Japan $(n=4)$, Korea $(n=3)$, China $(n=2)$, India $(n=1)$, Taiwan $(n=1)$, and Thailand $(n=1)$ were asked to perform a review of 16 whole slide images using a web browser-based image viewer or CaseViewer (3DHistech) installed on a personal computer. Observers were blinded to clinicopathologic data and molecular status. They were asked to interpret histologic features of 16 cases.

\section{Evaluation of histological features}

Histologic parameters and scoring scheme are summarized in Table 1. The following eight histologic parameters of nuclear features were semi-quantitatively scored from 0 (none) to 3 (frequent) (Fig. 1): nuclear enlargement, nuclear crowding/overlapping, nuclear elongation, irregular contour of the nuclear membrane, nuclear grooves, chromatin clearing, sickle-shaped nuclei [21], and NPIs. The sum of all these scores generated a total nuclear score ranging from 0 to 24 . In addition, reviewers were asked to classify each case as $B R A F$-like or $R A S$-like based on personal judgment of the histologic features irrespective of the total score.

\begin{tabular}{|c|c|}
\hline Histologic feature & Semiquantitative grading \\
\hline True papillae & Absent or present \\
\hline \multicolumn{2}{|l|}{ Nuclear features } \\
\hline 1. Nuclear enlargement & 0 : none \\
\hline 2. Nuclear crowding/overlapping & 1 : present in $<10 \%$ of tumor cells \\
\hline 3. Nuclear elongation & $\begin{array}{l}\text { 2: present in } 10 \%-50 \% \text { of tumor } \\
\text { cells }\end{array}$ \\
\hline 4. Irregular membrane contour & 3: present in $>50 \%$ of tumor cells \\
\hline \multicolumn{2}{|l|}{ 5. Nuclear grooves } \\
\hline \multicolumn{2}{|l|}{ 6. Chromatin clearing } \\
\hline \multicolumn{2}{|l|}{ 7. Sickle-shaped nuclei } \\
\hline \multirow[t]{4}{*}{ 8. Nuclear pseudoinclusions } & $0:$ none \\
\hline & 1: one or two in a whole slide \\
\hline & 2: three to five in a whole slide \\
\hline & 3: more than five in a whole slide \\
\hline Molecular type based on histology & $B R A F$-like or $R A S$-like \\
\hline
\end{tabular}

\section{Statistical analysis}

Fleiss' kappa ( $\kappa$ ) was calculated to measure the level of interobserver agreement. The strength of agreement was interpreted based on $\kappa$ value as follows: $<0.20$, poor; 0.21 to 0.40 , fair; 0.41 to 0.60 , moderate; 0.61 to 0.80 , good; and 0.81 to 1.00 , very good agreement. Mann-Whitney $U$ test was used to compare differences in histologic scores between two independent groups. All statistical analysis were performed using R software version 3.5.3 (www.r-project.org/) with “irr” package for Fleiss' $\kappa$ and GraphPad Prism version 6.05 (GraphPad Software, La Jolla, CA, USA) for all other statistical analyses. Statistical significance was considered when a two-sided $P$ value was less than 0.05 .

\section{RESULTS}

\section{Interpathologist agreement for interpretation of histologic features}

Semiquantitative results for all histologic features were grouped

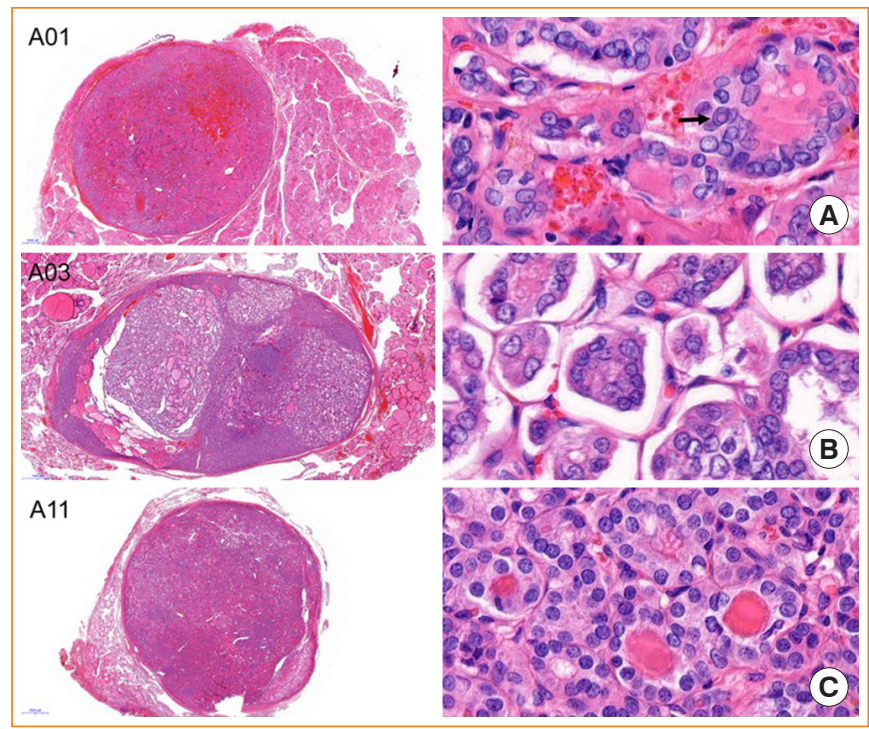

Fig. 1. Representative images of nuclear features of encapsulated follicular-patterned tumors. The right column shows high power views of the same tumor in the left column (H\&E stain, left, $\times 10$; right, $\times 1,000)$. (A) Case A01 with $B R A F^{\mathrm{V} 600 \mathrm{E}}$ shows fully developed nuclear features of papillary thyroid carcinoma, including nuclear pseudoinclusions (arrow). (B) In case A03 with $N R A S^{\mathrm{Q} 61 \mathrm{R}}$ mutation, a low power view shows a mixed microfollicular and macrofollicular pattern. Microfollicular areas reveal nuclear enlargement, nuclear crowding/overlapping, nuclear elongation, irregular contour of nuclear membrane, nuclear grooves, and chromatin clearing. (C) Case A11 with $N R A S^{\mathrm{Q} 61 \mathrm{R}}$ mutation shows small round dark and enlarged ovoid pale nuclei. 
into binary variables for statistical analysis. Fleiss' $\kappa$ was computed to assess the interpathologist agreement among 12 pathologists in interpreting histologic features of 16 follicular-patterned tumors (Table 2 ). There was a consistently poor agreement among 12 pathologists for true papillae $(\kappa=0.111)$, nuclear enlargement $(\kappa=0.085)$, nuclear crowding/overlapping $(\kappa=$ 0.162 ), nuclear elongation ( $\kappa=0.149)$, irregular nuclear membrane contour $(\kappa=0.149)$, chromatin clearing $(\kappa=0.180)$, and sickle-shaped nuclei $(\kappa=0.104)$. The agreement was fair for NPIs $(\kappa=0.214)$, total nuclear score $(\kappa=0.213)$, and molecular type (BRAF-like or $R A S$-like) based on histology $(\kappa=0.265)$.

\section{Correlation between molecular type and total score of nuclear features}

Sixteen tumors included two (A01 and A16) cases of $B R A F^{\mathrm{V} 600 \mathrm{E}}$ mutation, one (A02) case of $B R A F^{\mathrm{K} 601 \mathrm{E}}$, and 13 cases of $R A S$ mutations. The median value of total nuclear scores among 12 pathologists was 14 for two cases with $B R A F^{\mathrm{V} 600 \mathrm{E}}$ mutation and six for 14 cases with $R A S$-like mutations (Fig. 2). Therefore, we set a cut-off of total nuclear score at 14 which was used to ana-

Table 2. Interobserver Agreement among 12 Pathologists for Interpretation of Histologic Interpretation of 16 Encapsulated FollicularPatterned Thyroid Tumors

\begin{tabular}{|c|c|c|c|c|}
\hline Variable & Fleiss’ $\kappa$ & Strength of agreement & Z & $P$ value \\
\hline True papillae (absent vs. present) & 0.111 & Poor & 3.59 & $<0.001$ \\
\hline Nuclear enlargement ( $<10 \%$ vs. $>10 \%$ of tumor cells) & 0.085 & Poor & 2.77 & 0.006 \\
\hline Nuclear crowding/overlapping ( $<10 \%$ vs. $>10 \%$ of tumor cells) & 0.162 & Poor & 5.26 & $<0.001$ \\
\hline Nuclear elongation (<10\% vs. $>10 \%$ of tumor cells) & 0.149 & Poor & 4.83 & $<0.001$ \\
\hline Irregular contour of nuclear membrane ( $<10 \% \mathrm{vs} .>10 \%$ of tumor cells) & 0.073 & Poor & 2.38 & 0.018 \\
\hline Nuclear grooves $(<10 \%$ vs. $>10 \%$ of tumor cells $)$ & 0.054 & NA & 1.75 & 0.080 \\
\hline Chromatin clearing ( $<10 \%$ vs. $>10 \%$ of tumor cells) & 0.180 & Poor & 5.85 & $<0.001$ \\
\hline Sickle-shaped nuclei (<10\% vs. > $10 \%$ of tumor cells) & 0.104 & Poor & 3.38 & $<0.001$ \\
\hline Nuclear pseudoinclusions (absent vs. present) & 0.214 & Fair & 6.95 & $<0.001$ \\
\hline Sum of all scores of nuclear features ( <14 vs. $\geq 14)$ & 0.213 & Fair & 6.94 & $<0.001$ \\
\hline Molecular type based on histology (BRAF-like vs. RAS-like) & 0.265 & Fair & 8.61 & $<0.001$ \\
\hline
\end{tabular}

NA, not accessible.
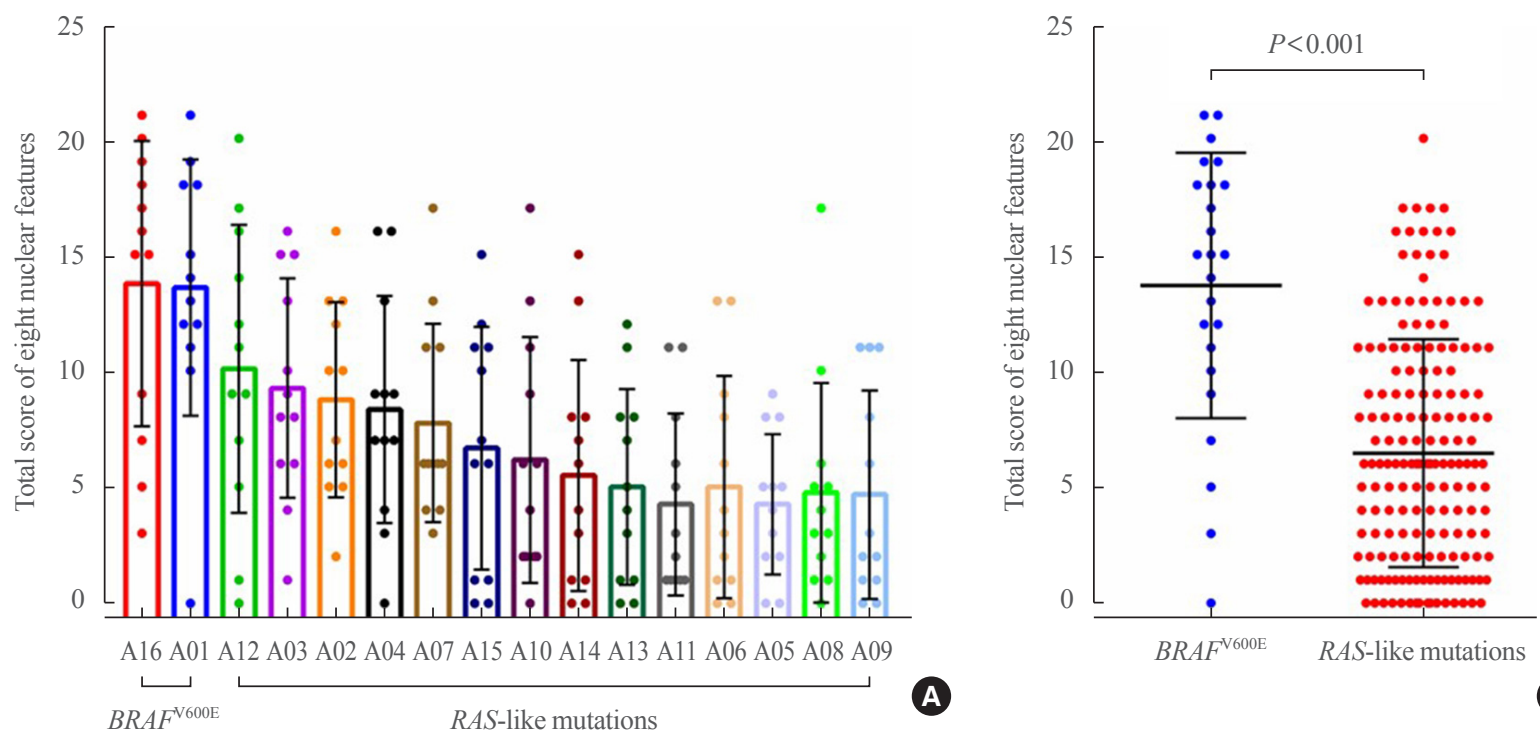

B

Fig. 2. (A, B) Distribution of total scores of eight histologic nuclear features assessed by 12 pathologists in 16 encapsulated follicular-patterned thyroid tumors. Total nuclear scores are significantly higher for cases with $B R A F^{\mathrm{V} 600 \mathrm{E}}$ than for those with $R A S$-like mutations. 
lyze interpathologist agreement (Table 2).

\section{Subgroup analysis of pathologists by threshold for interpreting nuclear features}

To determine interpathologist variability of thresholds in the assessment of eight nuclear features, nuclear scores for each case were combined to obtain a cumulative score for all 16 cases. $\mathrm{Cu}$ mulative scores for each pathologist ranged widely from 36 to 230 as shown in Fig. 3. These pathologists were categorized into three groups using these cumulative scores. Top three pathologists $(\mathrm{A}-\mathrm{C})$ scored cases with an overall higher total score ranging from 143 to 230 than the rest pathologists. Bottom three pathologists (J-L) had a tendency toward having a lower total score ranging from 36 to 79 (Fig. 3). Of four Japanese pathologists, three belonged to the intermediate group and one was in the lower group. However, two of three Korean pathologists belonged to the upper group and one was in the intermediate group. Of the three who were in the upper group, the other was Chinese.

After excluding these upper and lower groups in the sum of total nuclear scores, we further analyzed interpathologist agreement in the interpretation of histologic features among the remaining six pathologists. When data from six outliers (A, B, C, $\mathrm{J}, \mathrm{K}, \mathrm{L}$ ) were removed from analysis, kappa values increased for nuclear enlargement (from 0.085 to 0.297 ), nuclear crowding/ overlapping (from 0.162 to 0.363 ), irregular contour of the nuclear membrane (from 0.073 to 0.234 ), nuclear grooves (from 0.054 to 0.270 ), chromatin clearing (from 0.180 to 0.234 ), NPIs (from 0.214 to 0.508 ), sum of all scores (from 0.213 to 0.448 ), and molecular type based on histology (from 0.265 to 0.513 ) as shown in Table 3. The strength of agreement among these six pathologists was the best for detecting NPIs $(\kappa=0.508$, moderate agreement) but the worst for true papillae $(\kappa=0.057, P=0.378)$.

\section{Association between total nuclear score and correct answer rate for molecular type}

To analyze whether the six pathologists (D-I) correctly recognized molecular types, we compared their answers for each case with corresponding mutational status. All pathologists correctly interpreted two cases (A16 and A01) with $B R A F^{\mathrm{V} 600 \mathrm{E}}$ mutation as $B R A F$-like tumors and eight cases $(8 / 14)$ as $R A S$-like tumors (Fig. 4). When the sum of total nuclear scores was compared, cases with high and low nuclear scores had a high concordance rate whereas cases with intermediate nuclear scores (cases A12, A03, A02, and A04) had a low concordance rate (Fig. 4).

\section{Prediction of molecular types by nuclear features}

For six pathologists who were relatively consistent with each other without being biased on either extreme for the interpreta-

\begin{tabular}{|c|c|c|c|c|c|c|c|c|c|c|c|c|c|c|c|c|c|c|c|c|c|c|c|c|c|c|c|c|c|c|c|c|c|}
\hline \multirow[b]{2}{*}{ Observer } & \multicolumn{2}{|c|}{ A16 } & \multicolumn{2}{|c|}{ A01 } & \multicolumn{2}{|c|}{ A12 } & \multicolumn{2}{|c|}{ A03 } & \multicolumn{2}{|c|}{ A02 } & \multicolumn{2}{|c|}{ A04 } & \multicolumn{2}{|c|}{$\mathrm{A} 07$} & \multicolumn{2}{|c|}{ A15 } & \multicolumn{2}{|c|}{ A10 } & \multicolumn{2}{|c|}{ A14 } & \multicolumn{2}{|c|}{ A13 } & \multicolumn{2}{|c|}{ A11 } & \multicolumn{2}{|c|}{ A06 } & \multicolumn{2}{|c|}{ A05 } & \multicolumn{2}{|c|}{ A08 } & \multicolumn{2}{|c|}{ A09 } & \multirow[b]{2}{*}{ Sum } \\
\hline & s) & & & ర్ & 8 & & $<$ & \$ิ & of & & & \$े & & s5 & & $\frac{5}{6}$ & & $\frac{5}{6}$ & & $0_{0}^{5}$ & & & & है & & & & & & & $\gtrless$ & & \\
\hline A & 17 & BL & 19 & BL & 16 & RL & 16 & BL & 13 & RL & 16 & BL & 17 & RL & 15 & RL & 13 & BL & & BL & 12 & RL & 11 & RL & 13 & RL & & RL & 17 & RL & 11 & RL & 230 \\
\hline B & 18 & BL & 21 & BL & 17 & BL & 15 & RL & 16 & BL & 13 & RL & 13 & RL & 11 & RL & 11 & $B L$ & 15 & $B L$ & 11 & RL & 8 & RL & 13 & RL & 8 & RL & 10 & $\mathbf{R L}$ & 11 & RL & 209 \\
\hline C & 15 & BL & 10 & RL & 12 & BL & 13 & BL & 6 & RL & 9 & RL & 4 & RL & 10 & RL & 17 & BL & & RL & 8 & RL & 6 & RL & 9 & RL & 8 & RL & 0 & $\mathbf{R L}$ & 8 & RL & 143 \\
\hline D & 21 & BL & 18 & BL & 9 & RL & 9 & RL & 12 & RL & 7 & RL & 6 & RL & 11 & RL & 6 & RL & & RL & 5 & RL & 1 & RL & 8 & RL & & RL & 4 & RL & 6 & RL & 135 \\
\hline E & 20 & BL & 14 & BL & 20 & BL & 8 & BL & 5 & BL & 16 & BL & 11 & BL & 12 & BL & 4 & RL & & RL & 1 & RL & 11 & RL & 1 & RL & $?$ & RL & 1 & $\mathbf{R L}$ & 0 & RL & 130 \\
\hline $\mathbf{F}$ & 19 & BL & 12 & $B L$ & 11 & RL & 15 & $B L$ & 7 & RL & 9 & BL & 11 & RL & 6 & RL & 6 & RL & & RL & 7 & & 1 & RL & 6 & RL & 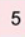 & RL & 5 & RL & 1 & RL & 128 \\
\hline G & 16 & BL & 18 & BL & 14 & RL & 8 & RL & 13 & RL & 7 & RL & 6 & RL & 7 & RL & 9 & RL & & RL & 8 & RL & 5 & RL & 0 & RL & 0 & RL & 3 & RL & 3 & RL & 123 \\
\hline $\mathrm{H}$ & 15 & $B L$ & 15 & BL & 5 & BL & 10 & $B L$ & 10 & BL & 9 & BL & 6 & RL & 1 & RL & 2 & RL & & RL & 1 & RL & 1 & $\mathrm{KL}$ & 2 & RL & & RL & 1 & RL & 1 & RL & 85 \\
\hline I & 7 & BL & 13 & BL & 7 & BL & 4 & RL & 6 & RL & 4 & RL & 6 & RL & 6 & RL & 2 & RL & & RL & 4 & RL & 3 & RL & 4 & RL & 5 & RL & 5 & RL & 2 & RL & 81 \\
\hline$J$ & 9 & RL & 12 & BL & 0 & RL & 6 & RL & 10 & RL & 7 & BL & 6 & BL & 0 & RL & 2 & RL & & RL & 3 & RL & 2 & RL & 3 & RL & 2 & RL & 6 & $\mathbf{R L}$ & 11 & RL & 79 \\
\hline $\mathrm{K}$ & 5 & RL & 11 & RL & 9 & BL & 1 & RL & 2 & RL & 0 & RL & 3 & RL & 0 & RL & 0 & RL & & RL & 0 & RL & 1 & RL & 0 & RL & 0 & RL & 2 & $\mathbf{R L}$ & 0 & RL & 34 \\
\hline $\mathbf{L}$ & 3 & RL & 0 & RL & 1 & RL & 6 & RL & 5 & RL & 3 & RL & 4 & $\mathbf{R L}$ & 1 & RL & 2 & $R$ & & RL & 0 & & 1 & RL & 1 & RL & & RL & 3 & RL & 2 & RL & 36 \\
\hline
\end{tabular}

Fig. 3. Heatmap illustrating variations of nuclear scores and molecular types among 12 pathologists (A-L) in 16 encapsulated follicular-patterned thyroid tumors. Each pathologist blinded to molecular data assessed whether tumors were BRAF-like (BL) or RAS-like (RL) based on their histologic features. The number in each cell means the sum of the grading score of eight nuclear histologic features. Values in the farright column of the table mean the sum of the nuclear scores of 16 cases for each pathologist. All pathologists are listed in order according to their sum of nuclear scores. In the far-left column, three pathologists marked in blue had a tendency to give high scores and over-interpret histologic nuclear features whereas three pathologists marked in orange had a tendency to give low scores and underestimate degrees of nuclear changes. 
Table 3. Interobserver Agreement of Six Experts for the Histologic Interpretation of Encapsulated Follicular-Patterned Thyroid Tumors

\begin{tabular}{|c|c|c|c|c|}
\hline Variable & Fleiss' $\kappa$ & Strength of agreement & Z & $P$ value \\
\hline True papillae & 0.057 & NA & 0.88 & 0.378 \\
\hline Nuclear enlargement ( $<10 \%$ vs. $>10 \%$ of tumor cells) & 0.297 & Fair & 4.60 & $<0.001$ \\
\hline Nuclear crowding/overlapping ( $<10 \%$ vs. $>10 \%$ of tumor cells) & 0.363 & Fair & 5.62 & $<0.001$ \\
\hline Nuclear elongation ( $<10 \%$ vs. $>10 \%$ of tumor cells) & 0.131 & Poor & 2.03 & 0.043 \\
\hline Irregular contour of nuclear membrane ( $<10 \%$ vs. $>10 \%$ of tumor cells) & 0.234 & Fair & 3.62 & $<0.001$ \\
\hline Nuclear grooves ( $<10 \%$ vs. $>10 \%$ of tumor cells $)$ & 0.270 & Fair & 4.19 & $<0.001$ \\
\hline Chromatin clearing ( $<10 \%$ vs. $>10 \%$ of tumor cells) & 0.234 & Fair & 3.62 & $<0.001$ \\
\hline Sickle-shaped nuclei ( $<10 \%$ vs. $>10 \%$ of tumor cells) & 0.105 & Poor & 1.63 & 0.103 \\
\hline Nuclear pseudoinclusions (absent vs. present) & 0.508 & Moderate & 7.87 & $<0.001$ \\
\hline Sum of all scores $(<14$ vs. $\geq 14)$ & 0.448 & Moderate & 6.95 & $<0.001$ \\
\hline Molecular type ( $B R A F$-like vs. $R A S$-like) based on histology & 0.513 & Moderate & 7.95 & $<0.001$ \\
\hline PTC-type nuclear features & 0.233 & Fair & 3.62 & $<0.001$ \\
\hline
\end{tabular}

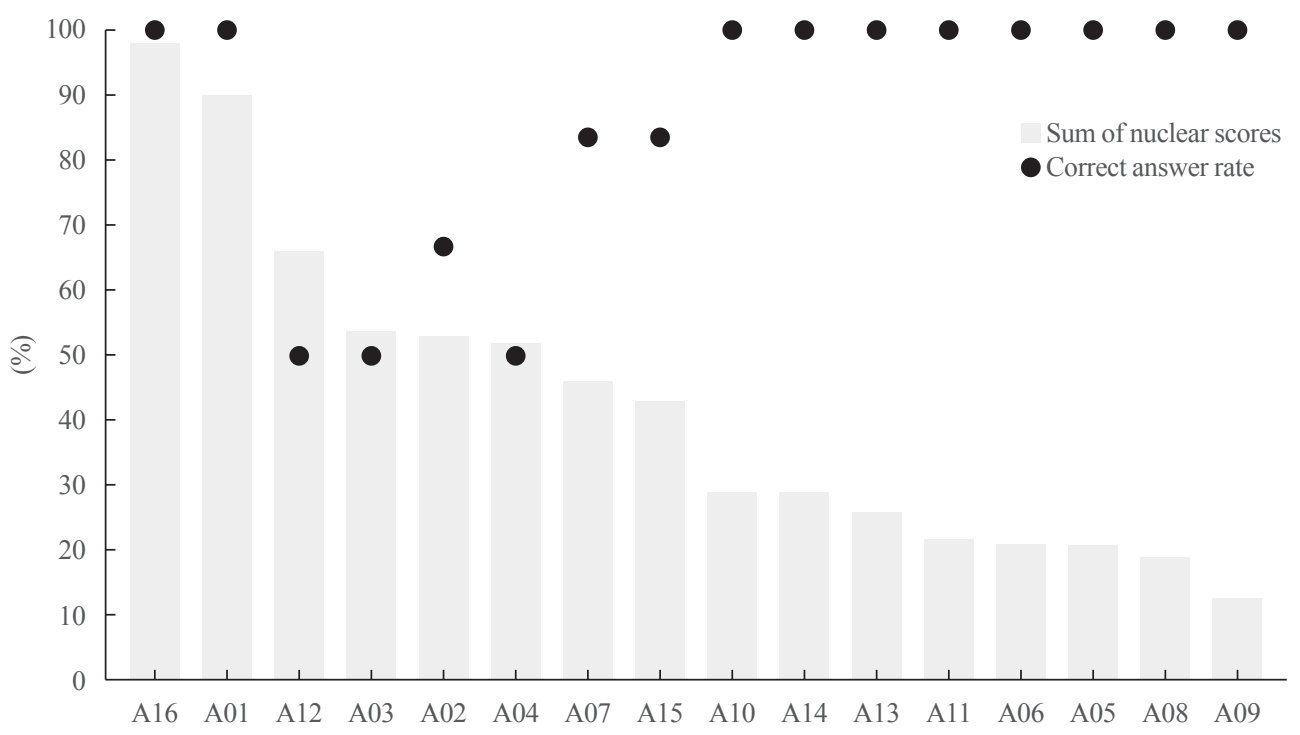

Fig. 4. Association between the sum of total nuclear scores and correct answer rate for molecular type by six pathologists. Nuclear scores assessed by six pathologists were summed up to denote the sum of nuclear scores on the Y-axis on the left side of the graph. Of 16 encapsulated follicular-patterned thyroid tumors, two cases A16 and A01 had $B R A F^{\mathrm{V} 600 \mathrm{E}}$ mutation and the remaining 14 cases had $R A S$-like mutations. All six pathologists interpreted whether each tumor was $B R A F$-like or $R A S$-like based on its histologic features. They correctly answered molecular types for 10/16 cases (case A16, A01, A10, A14, A13, A11, A06, A05, A08, and A09).

tion of histologic features, we conducted a further analysis to identify histologic features that could predict mutational status.

Histologic features and total nuclear scores for each case were correlated with mutational patterns (Fig. 5). Nuclear scores for eight histologic features were higher for cases with $B R A F^{\mathrm{V} 600 \mathrm{E}}$ mutation than for cases with $R A S$-like mutations (Fig. 5). As a result, the sum of total nuclear scores was significantly higher for cases with $B R A F^{\mathrm{V} 600 \mathrm{E}}$ mutation than for cases with $R A S$-like mutations $(P<0.001)$. Scores of NPIs were detected in two cases with $B R A F^{\mathrm{V} 600 \mathrm{E}}$ mutation and in five cases with $R A S$-like mutations (Fig. 5). However, a thorough review of whole slide images revealed that nuclear vacuoles or bubbles were misinterpreted as NPIs in five of 14 tumors with $R A S$-like mutations (Fig. 6). There were no true NPIs in tumors with $R A S$-like mutations. True NPIs were found at more than 20 cells within a whole slide image in tumors with $B R A F^{\mathrm{V} 600 \mathrm{E}}$ mutation. 


\begin{tabular}{|c|c|c|c|c|c|c|c|c|c|c|c|c|c|c|c|c|}
\hline Case \# & A16 & $\mathrm{A} 01$ & A12 & $\mathrm{A} 03$ & $\mathrm{~A} 02$ & $\mathrm{~A} 04$ & $\mathrm{~A} 07$ & $\mathrm{~A} 15$ & A10 & A14 & A13 & A11 & $\mathrm{A} 06$ & A05 & $\mathrm{A} 08$ & $\mathrm{~A} 09$ \\
\hline Histologic features & 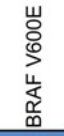 & 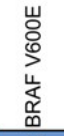 & 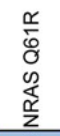 & $\begin{array}{l}\frac{\alpha}{\delta} \\
\varnothing \\
\frac{1}{\alpha} \\
\frac{\alpha}{z}\end{array}$ & 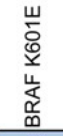 & $\begin{array}{l}\frac{\alpha}{\delta} \\
\varnothing \\
\frac{\infty}{\alpha} \\
\frac{\alpha}{z}\end{array}$ & 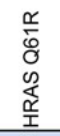 & $\begin{array}{l}\frac{y}{\delta} \\
\varnothing \\
0 \\
\frac{1}{\alpha} \\
\frac{\alpha}{z}\end{array}$ & 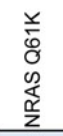 & $\begin{array}{l}\frac{x}{\delta} \\
8 \\
0 \\
\frac{\alpha}{\alpha} \\
\frac{1}{z}\end{array}$ & $\begin{array}{l}\frac{x}{5} \\
8 \\
0 \\
\frac{1}{\alpha} \\
\frac{\alpha}{z}\end{array}$ & 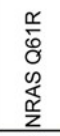 & 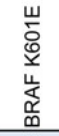 & 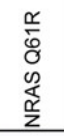 & 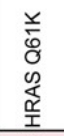 & $\begin{array}{l}\frac{y}{\delta} \\
\varnothing \\
0 \\
\frac{1}{\alpha} \\
\frac{\alpha}{z}\end{array}$ \\
\hline Nuclear enlargement & 17 & 16 & 12 & 12 & 11 & 9 & 9 & 10 & 8 & 4 & 8 & 6 & 8 & 6 & 5 & 5 \\
\hline Nuclear crowding-overlapping & 15 & 14 & 9 & 11 & 7 & 8 & 7 & 7 & 7 & 7 & 3 & 4 & 2 & 2 & 1 & 2 \\
\hline Nuclear elongation & 13 & 11 & 7 & 6 & 6 & 7 & 4 & 7 & 4 & 5 & 3 & 0 & 3 & 2 & 3 & 2 \\
\hline Irregular contours & 11 & 12 & 9 & 7 & 9 & 9 & 7 & 7 & 3 & 5 & 4 & 4 & 4 & 4 & 4 & 2 \\
\hline Nuclear grooves & 12 & 10 & 7 & 5 & 7 & 5 & 7 & 5 & 2 & 4 & 4 & 4 & 3 & 3 & 2 & 2 \\
\hline Chromatin clearing & 13 & 10 & 11 & 10 & 8 & 9 & 9 & 3 & 4 & 3 & 2 & 3 & 0 & 1 & 0 & 0 \\
\hline Sickle-shaped nuclei & 9 & 5 & 5 & 3 & 5 & 4 & 3 & 3 & 1 & 1 & 2 & 1 & 1 & 2 & 3 & 0 \\
\hline Nuclear pseudoinclusions & 8 & 12 & 6 & 0 & 0 & 1 & 0 & 1 & 0 & 0 & 0 & 0 & 0 & 1 & 1 & 0 \\
\hline Sum of all socres & 98 & 90 & 66 & 54 & 53 & 52 & 46 & 43 & 29 & 29 & 26 & 22 & 21 & 21 & 19 & 13 \\
\hline
\end{tabular}

Fig. 5. Heatmap illustrating the distribution of total score of each histologic feature assessed by six pathologists selected (D-I) for 16 encapsulated follicular-patterned thyroid tumors. The number in each cell means the sum of points grading with each histologic feature interpreted by six pathologists.
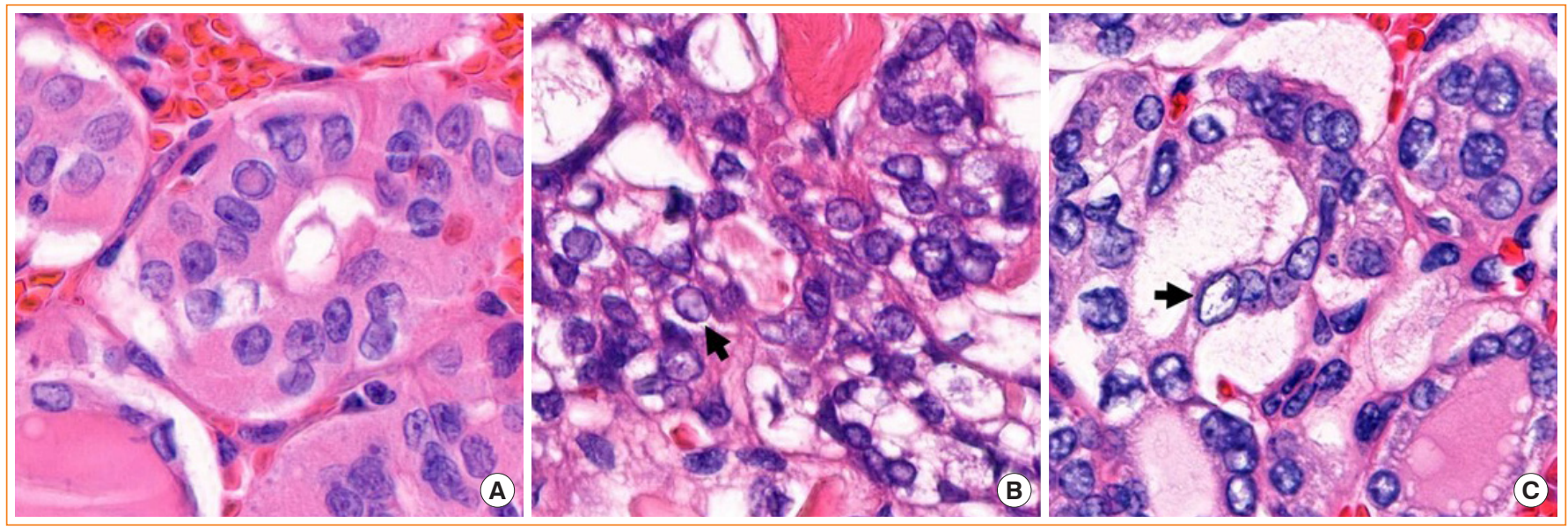

Fig. 6. Nuclear pseudoinclusions and nuclear vacuoles mimicking nuclear pseudoinclusions. (A) An intranuclear cytoplasmic pesudoinclusion found in case A01 contains a round eosinophilic intranuclear body well demarcated by a rim of dense nuclear membrane. The color and texture are the same as the surrounding cytoplasm. Mimics of nuclear pseudoinclusions in case 12 (B) and case 04 (C) showed pale chromatin and irregularly shaped empty-looking nuclear vacuoles that lacked color and texture of the cytoplasm (arrows). Nucleoli were found within the nuclear vacuoles $(H \& E$ stain, $\times 1,000)$.

\section{DISCUSSION}

Despite well-defined diagnostic criteria, the diagnosis of encapsulated follicular-patterned thyroid tumors remains elusive. Diagnostic difficulties and poor reproducibility are mostly attributed to differences in evaluating nuclear features of PTC and invasion of tumor capsule and/or blood vessels. In the present study, we focused on evaluating the interobserver agreement and morphological features to delineate encapsulated follicularpatterned PTC with $B R A F^{\mathrm{V} 600 \mathrm{E}}$ mutation from benign or borderline thyroid tumors with $R A S$ mutations. Results showed that NPIs and high nuclear score calculated by eight nuclear features could differentiate $B R A F^{\mathrm{V} 600 \mathrm{E}}$-mutated thyroid tumors with a predominant follicular architecture from thyroid tumors with $R A S$-like mutations. The interobserver agreement for the interpretation of NPIs and total nuclear scores was moderate among six pathologists of a subgroup for identifying these findings.

This study conducted two rounds of statistical analysis for the interobserver agreement in interpreting histologic features. In the first round, the agreement in interpreting histologic features among 12 pathologists was generally poor except for the interpretation of NPIs and total nuclear score. These results showed a wide variation in diagnostic thresholds and interpretation for a wide spectrum of encapsulated follicular-patterned thyroid tu- 
mors among endocrine pathologists. As the present study aimed to identify nuclear features to differentiate $B R A F^{\mathrm{V} 600 \mathrm{E}}$-mutated thyroid tumors from those with $R A S$-like mutations, we excluded experts with outlying scores. In the second round, statistical analysis was performed for a group of six pathologists. The overall agreement in interpreting histologic features was increased. The molecular type was more correctly interpreted for cases with high and low total nuclear scores determined based on eight nuclear features. The agreement among pathologists was higher for the evaluation of NPIs and total nuclear scores compared to that for other nuclear features.

NPIs as a characteristic of PTC can be also seen in a variety of other thyroid tumors such as medullary thyroid carcinoma and hyalinizing trabecular tumor. However, it is very rarely seen in benign thyroid nodules. Although the presence of NPIs is not an exclusion criterion for the diagnosis of NIFTP, NPIs are rarely found or absent in cytology specimens of NIFTP [22,23]. It is known that NPIs are easier to identify in cytology slides than in histopathology slides because nuclear vacuoles or bubbles mimicking NPIs are more frequently seen in the surgical specimens than in cytology slides [24].

NPIs are round and regular. Their inclusion borders are sharply defined so that inclusions containing cytoplasm can be clearly distinguished from surrounding nuclear chromatin texture. Contents of NPIs should appear similar to the cytoplasm of the tumor cells, as shown in Fig. 6. However, NPIs may be missed or misinterpreted because they are not always seen even in classic PTC and nuclear vacuoles mimicking NPIs are frequently seen. Therefore, the principal investigator performed a meticulous review of whole slide images on high power to distinguish true NPIs from their mimics in all 16 cases. This thorough review revealed that two cases with $B R A F^{\mathrm{V} 600 \mathrm{E}}$ mutation had true NPIs whereas other $R A S$-like tumors contained only nuclear vacuoles mimicking NPIs (Fig. 6). Nuclear vacuoles or bubbles were found in every $R A S$-like tumor at varying degrees, leading to misinterpretation as NPIs. Tumors with $B R A F^{\mathrm{V} 600 \mathrm{E}}$ had more than 20 cells with NPIs within one slide. However, some disagreement occurred among pathologists regarding the identification of NPIs. The overall agreement for the detection of NPIs among six pathologists was moderate. NPIs were overcalled in five of $14 R A S$-like tumors by at least one pathologist. Two pathologists could not identify NPIs in two BRAF-like tumors. Lack of concentration while reviewing virtual slides might have contributed to missed detection of NPIs. Nevertheless, since the interobserver agreement was higher for the assessment of NPIs than for other nuclear features, the presence of NPIs might be the most useful histologic feature to rule-in $B R A F$-like tumors. Therefore, to achieve a highly specific diagnosis of $B R A F$-like PTC, pathologists should be more cautious when interpreting NPIs on histopathology slides [25,26].

Although not pathognomonic, the appearance of true papillae in thyroid tumors should prompt a careful search for characteristic nuclear features of PTC. According to the revised criteria for NIFTP, the presence of well-formed papillae with a fibrovascular core is an exclusion criterion for NIFTP [5]. Since NIFTP can show simple slender papillae without a fibrovascular core and papillary infoldings resembling Sanderson's polsters, true papillae should be differentiated from hyperplastic-type papillary structure with rudimentary fibrovascular cores $[5,27]$. Hyperplastic-type papillae seen in NIFTP are lined by follicular cells, showing less developed nuclear features of PTC. However, true papillae in classic PTC are lined by cells with well-developed nuclear features. Even single true papilla is not allowed for the diagnosis of NIFTP per the diagnostic criteria. In real practice, however, histologic features of papillae are often inconsistent from tumor to tumor. Discrepancies exist in the application of the subjective criteria among pathologists. In our study, pathologists tended to detect true papillae more frequently for cases with higher nuclear scores. Similarly, the presence of papillae was interpreted less frequently for cases with lower nuclear scores. However, the interobserver agreement was lower for the evaluation of true papillae than for other nuclear features. Therefore, true papillae might be of diagnostic help only when they are interpreted with strict criteria.

Nuclear features of PTC are readily identifiable in most PTCs. However, some tumors show subtle nuclear changes. Nuclear features can be found focally within the tumor. In this respect, the nuclear scoring system can help achieve reproducibility among pathologists in the diagnosis of PTC. A threepoint nuclear scoring system has been used for the diagnosis of NIFTP and PTC since 2016 [4]. This three-point nuclear score is interpreted as the presence (score of $0-1$ ) or absence (score of 2-3) of nuclear features to diagnose NIFTP by an assigned score of 0 (absent) or 1 (present) for each category. In an international study performed by American, Japanese, and British pathologists, a substantial agreement $(\kappa=0.60-0.64)$ was achieved for the diagnosis of NIFTP [28]. In a recent Asian study that reviewed web-based virtual slides by our working group, the interobserver agreement was moderate for the diagnosis of NIFTP using the same three-point nuclear score [3]. However the nuclear score could not differentiate $B R A F$-like tumors from $R A S$-like tumors [3]. As the three-point nuclear 
score is a system that interprets whether each category is present or absent within the tumor, diagnosis is not affected by the amount or the percentage for each scored parameter found in the tumor. On the other hand, when we evaluated total nuclear score for eight nuclear features based on the degree of nuclear changes and their distribution within the tumor, total nuclear score with a range of 0 to 24 could differentiate $B R A F$-like tumors from $R A S$-like tumors using a cut-off value of score 14 .

Multinucleated giant cells are found in various thyroid lesions including Hashimoto's thyroiditis, granulomatous thyroiditis, palpation thyroiditis, and PTC [29]. Some studies have reported that multinucleated giant cells are rarely found in NIFTP and follicular adenoma $[22,30]$. Thus, the principal investigator of this study additionally evaluated the presence of multinucleated giant cells. Multinucleated giant cells were identified in all cases with $B R A F^{\mathrm{V} 600 \mathrm{E}}$ mutation and in one of 14 cases with $R A S$-like mutations. Although the presence of multinucleated giant cells has no diagnostic specificity for thyroid lesions, results of the present study are similar to previous studies showing the absence of multinucleated giant cells in NIFTP and follicular adenoma $[22,30]$. Other previous studies have shown that the presence of multinucleated giant cells is related to extrathyroidal extension and lymph node metastasis in patients with PTC [29,31]. Therefore, the appearance of multinucleated giant cells in cytologic and histologic slides should raise the possibility of a diagnosis of PTC.

The status of $B R A F^{\mathrm{V} 600 \mathrm{E}}$ mutation can be reliably detected on histologic section by immunohistochemistry [32-34]. Several studies have shown that $B R A F^{\mathrm{V} 600 \mathrm{E}}$ (VE1) immunostaining can identify $B R A F^{\mathrm{V} 600 \mathrm{E}}$ mutation in small size samples, including those with low cellularity and cases with a low mutant allele frequency [32-34]. Because most mutations of NRAS, HRAS, and $K R A S$ genes occur at multiple positions of codons 12, 13, and 61, immunohistochemistry cannot cover all types of RAS mutations. Testing of RAS mutations should be done using molecular techniques. VE1 immunostaining can be particularly helpful in diagnosing PTC for cases with a high total nuclear score and/or NPIs.

Since noninvasive encapsulated thyroid tumors with predominant follicular growth and $B R A F^{\mathrm{V} 600 \mathrm{E}}$ are rarely found, particularly in Asian institutions [13,35], our study was limited by the small number of cases. Moreover, additional validation study was not performed. Another limitation was that observers might lack concentration while they reviewed slides as two pathologists detected no NPIs in case A01 in which there were at least 30 NPIs confirmed by the lead pathologist. This study was con- ducted using virtual slide images. It might have affected the interpretation by some pathologists who were not familiar with reviewing digital slides. Nevertheless, we minimized bias by excluding the outliers. Molecular testing data served as reference standard. Mutation-positive cases were only used to identify histologic features to predict molecular type. As whole slide images were used in the present study, we could evaluate the quantitative area within a tumor that showed eight nuclear features and created a total nuclear score with a range from 0 to 24 . To the best of our knowledge, this is the first study to compare total nuclear score and molecular type in encapsulated follicularpatterned tumors. We also analyzed different nuclear parameters by their potential values in differential diagnosis of $B R A F^{\mathrm{V} 600 \mathrm{E}}$ like and $R A S$-like tumors and chose NPI as the best candidate.

In summary, encapsulated follicular-patterned tumors with nuclear features of PTC should be carefully examined for the presence of NPIs. NPIs and high nuclear scores assessed by eight nuclear features were hallmarks of $B R A F$-like tumors with predominant follicular growth, showing a moderate strength of agreement for their identification among Asian pathologists. However, relaxation of rigid criteria for nuclear features of PTC by some pathologists resulted in overdiagnosis of PTC. Thus, testing for $B R A F^{\mathrm{V} 600 \mathrm{E}}$ might be a useful adjunct in the diagnosis of encapsulated PTC, especially in cases with a high total nuclear score.

\section{CONFLICTS OF INTEREST}

No potential conflict of interest relevant to this article was reported.

\section{ACKNOWLEDGMENTS}

We would like to thank Dr. Deepali Jain (All India Institute of Medical Sciences, New Delhi, India) for providing her expert opinion. This research was supported by a grant (NRF-2020R1F1A1070028) from the Basic Science Research Program through the National Research Foundation of Korea funded by the Ministry of Science and ICT.

\section{AUTHOR CONTRIBUTIONS}

Conception or design: C.K.J., A.B., K.K. Acquisition, analysis, or interpretation of data: C.K.J., A.B., D.E.S., J.H.K, Y.Z., Z.L., S.K., C.R.L., M.H., K.K., K.K. Drafting the work or revising: C.K.J., A.B., D.E.S., J.H.K, Y.Z., Z.L., S.K., C.R.L., M.H., 
K.K., K.K. Final approval of the manuscript: C.K.J., A.B., D. E.S., J.H.K, Y.Z., Z.L., S.K., C.R.L., M.H., K.K., K.K.

\section{ORCID}

Chan Kwon Jung https://orcid.org/0000-0001-6843-3708

\section{REFERENCES}

1. Lloyd RV, Osamura RY, Kloppel G, Rosai J. WHO classification of tumors of endocrine organs. 4th ed. Lyon: International Agency for Research on Cancer (IARC); 2017. p. 65-91.

2. Zhu Y, Li Y, Jung CK, Song DE, Hang JF, Liu Z, et al. Histopathologic assessment of capsular invasion in follicular thyroid neoplasms-an observer variation study. Endocr Pathol 2020;31:132-40.

3. Liu Z, Bychkov A, Jung CK, Hirokawa M, Sui S, Hong S, et al. Interobserver and intraobserver variation in the morphological evaluation of noninvasive follicular thyroid neoplasm with papillary-like nuclear features in Asian practice. Pathol Int 2019;69:202-10.

4. Nikiforov YE, Seethala RR, Tallini G, Baloch ZW, Basolo F, Thompson LD, et al. Nomenclature revision for encapsulated follicular variant of papillary thyroid carcinoma: a paradigm shift to reduce overtreatment of indolent tumors. JAMA Oncol 2016;2:1023-9.

5. Nikiforov YE, Baloch ZW, Hodak SP, Giordano TJ, Lloyd $\mathrm{RV}$, Seethala RR, et al. Change in diagnostic criteria for noninvasive follicular thyroid neoplasm with papillarylike nuclear features. JAMA Oncol 2018;4:1125-6.

6. Cancer Genome Atlas Research Network. Integrated genomic characterization of papillary thyroid carcinoma. Cell 2014;159:676-90.

7. Fagin JA, Wells SA Jr. Biologic and clinical perspectives on thyroid cancer. N Engl J Med 2016;375:1054-67.

8. Tirro E, Martorana F, Romano C, Vitale SR, Motta G, Di Gregorio S, et al. Molecular alterations in thyroid cancer: from bench to clinical practice. Genes (Basel) 2019;10:709.

9. Paulson VA, Shivdasani P, Angell TE, Cibas ES, Krane JF, Lindeman NI, et al. Noninvasive follicular thyroid neoplasm with papillary-like nuclear features accounts for more than half of "carcinomas" harboring RAS mutations. Thyroid 2017;27:506-11.

10. Kakudo K. Thyroid FNA cytology. 2nd ed. Singapore: Springer Nature Singapore Pte Ltd; 2019. Chapter 21, Nuclear features of papillary thyroid carcinoma (BRAF-like tu- mors), noninvasive follicular thyroid neoplasm with papillary-like nuclear features (RAS-like tumors), and follicular adenoma/follicular thyroid carcinoma (RAS-like tumors); p. 173-9.

11. Hirokawa M, Carney JA, Goellner JR, DeLellis RA, Heffess CS, Katoh R, et al. Observer variation of encapsulated follicular lesions of the thyroid gland. Am J Surg Pathol 2002;26:1508-14.

12. Rosai J. The encapsulated follicular variant of papillary thyroid carcinoma: back to the drawing board. Endocr Pathol 2010;21:7-11.

13. Bychkov A, Jung CK, Liu Z, Kakudo K. Noninvasive follicular thyroid neoplasm with papillary-like nuclear features in Asian practice: perspectives for surgical pathology and cytopathology. Endocr Pathol 2018;29:276-88.

14. Bychkov A, Keelawat S, Agarwal S, Jain D, Jung CK, Hong $\mathrm{S}$, et al. Impact of non-invasive follicular thyroid neoplasm with papillary-like nuclear features on the Bethesda system for reporting thyroid cytopathology: a multi-institutional study in five Asian countries. Pathology 2018;50:411-7.

15. Hirokawa M, Higuchi M, Suzuki A, Hayashi T, Kuma S, Miyauchi A. Prevalence and diagnostic significance of noninvasive follicular thyroid neoplasm with papillary-like nuclear features among tumors previously diagnosed as follicular adenoma: a single-institutional study in Japan. Endocr J 2020;67:1071-5.

16. Zajkowska K, Kopczynski J, Gozdz S, Kowalska A. Noninvasive follicular thyroid neoplasm with papillary-like nuclear features: a problematic entity. Endocr Connect 2020;9: R47-58.

17. Cho U, Mete O, Kim MH, Bae JS, Jung CK. Molecular correlates and rate of lymph node metastasis of non-invasive follicular thyroid neoplasm with papillary-like nuclear features and invasive follicular variant papillary thyroid carcinoma: the impact of rigid criteria to distinguish non-invasive follicular thyroid neoplasm with papillary-like nuclear features. Mod Pathol 2017;30:810-25.

18. Jung CK, Kim Y, Jeon S, Jo K, Lee S, Bae JS. Clinical utility of EZH1 mutations in the diagnosis of follicular-patterned thyroid tumors. Hum Pathol 2018;81:9-17.

19. Bychkov A, Kakudo K, Hong S. Current practices of thyroid fine-needle aspiration in Asia: a missing voice. J Pathol Transl Med 2017;51:517-20.

20. Jung CK, Hong S, Bychkov A, Kakudo K. The use of fineneedle aspiration (FNA) cytology in patients with thyroid nodules in Asia: a brief overview of studies from the Work- 
ing Group of Asian Thyroid FNA Cytology. J Pathol Transl Med 2017;51:571-8.

21. Rossi ED, Bizzarro T, Martini M, Capodimonti S, Fadda G, Larocca LM, et al. Morphological parameters able to predict BRAF(V600E) mutated malignancies on thyroid fine-needle aspiration cytology: our institutional experience. Cancer Cytopathol 2014;122:883-91.

22. Legesse T, Parker L, Heath J, Staats PN. Distinguishing non-invasive follicular thyroid neoplasm with papillary-like nuclear features (NIFTP) from classic and invasive follicular-variant papillary thyroid carcinomas based on cytologic features. J Am Soc Cytopathol 2019;8:11-7.

23. Mahajan S, Agarwal S, Kocheri N, Jain D, Mathur SR, Iyer VK. Cytopathology of non-invasive follicular thyroid neoplasm with papillary-like nuclear features: a comparative study with similar patterned papillary thyroid carcinoma variants. Cytopathology 2018;29:233-40.

24. Arora SK, Dey P. Intranuclear peudoinclusions: morphology, pathogenesis, and significance. Diagn Cytopathol 2012; 40:741-4.

25. Ip YT, Dias Filho MA, Chan JK. Nuclear inclusions and pseudoinclusions: friends or foes of the surgical pathologist? Int J Surg Pathol 2010;18:465-81.

26. Das DK. Intranuclear cytoplasmic inclusions in fine-needle aspiration smears of papillary thyroid carcinoma: a study of its morphological forms, association with nuclear grooves, and mode of formation. Diagn Cytopathol 2005;32:264-8.

27. Alves VA, Kakudo K, LiVolsi V, Lloyd RV, Nikiforov YE, Nose V, et al. Noninvasive follicular thyroid neoplasm with papillary-like nuclear features (NIFTP): achieving better agreement by refining diagnostic criteria. Clinics (Sao Paulo) 2018;73:e576.

28. Thompson LD, Poller DN, Kakudo K, Burchette R, Nikiforov YE, Seethala RR. An international interobserver variability reporting of the nuclear scoring criteria to diagnose noninvasive follicular thyroid neoplasm with papillary-like nuclear features: a validation study. Endocr Pathol 2018;29: 242-9.

29. Choi JE, Bae JS, Lim DJ, Jung SL, Jung CK. Atypical histiocytoid cells and multinucleated giant cells in fine-needle aspiration cytology of the thyroid predict lymph node metastasis of papillary thyroid carcinoma. Cancers (Basel) 2019;11:816.

30. Selvaggi SM. The presence of multinucleated giant cells: noninvasive follicular thyroid neoplasm with papillary-like nuclear features vs the follicular variant of papillary thyroid carcinoma. Diagn Cytopathol 2019;47:1007-10.

31. Brooks E, Simmons-Arnold L, Naud S, Evans MF, Elhosseiny A. Multinucleated giant cells' incidence, immune markers, and significance: a study of 172 cases of papillary thyroid carcinoma. Head Neck Pathol 2009;3:95-9.

32. Dvorak K, Aggeler B, Palting J, McKelvie P, Ruszkiewicz A, Waring P. Immunohistochemistry with the anti-BRAF V600E (VE1) antibody: impact of pre-analytical conditions and concordance with DNA sequencing in colorectal and papillary thyroid carcinoma. Pathology 2014;46:509-17.

33. Singarayer R, Mete O, Perrier L, Thabane L, Asa SL, Van Uum $\mathrm{S}$, et al. A systematic review and meta-analysis of the diagnostic performance of BRAFV600E immunohistochemistry in thyroid histopathology. Endocr Pathol 2019;30: 201-18.

34. Choden S, Keelawat S, Jung CK, Bychkov A. VE1 immunohistochemistry improves the limit of genotyping for detecting BRAFV600E mutation in papillary thyroid cancer. Cancers (Basel) 2020;12:596.

35. Bychkov A, Hirokawa M, Jung CK, Liu Z, Zhu Y, Hong $\mathrm{SW}$, et al. Low rate of noninvasive follicular thyroid neoplasm with papillary-like nuclear features in Asian practice. Thyroid 2017;27:983-4. 